\title{
Efficient and Effective Quality Assessment of As-Is Building Information Models and 3D Laser-Scanned Data
}

\author{
Pingbo Tang ${ }^{1}$, Engin Burak Anil ${ }^{2}$, Burcu Akinci ${ }^{2}$, Daniel Huber ${ }^{3}$ \\ ${ }^{1}$ Civil and Construction Engineering Department, Western Michigan University, 4601 \\ Campus Drive, Kalamazoo, MI 49008-5316; PH (269) 276-3203; FAX (269) \\ 276-3211; email: pingbo.tang@wmich.edu \\ ${ }^{2}$ Civil and Environmental Engineering Department, Carnegie Mellon University, \\ 5000 Forbes, Pittsburgh, PA 15213-3890; PH (412) 268-2959; FAX (412) 268-7813; \\ email: \{eanil, bakinci\}@ andrew.cmu.edu \\ ${ }^{3}$ Robotics Institute, Carnegie Mellon University, 5000 Forbes Avenue, Pittsburgh, PA \\ 15213; PH (412) 268-2991; FAX (412) 268-6436; email: dhuber@cs.cmu.edu
}

\begin{abstract}
Documenting as-is conditions of buildings using 3D laser scanning and Building Information Modeling (BIM) technology is being adopted as a practice for enhancing effective management of facilities. Many service providers generate as-is BIMs based on laser-scanned data. It is necessary to conduct timely and comprehensive assessments of the quality of the laser-scanned data and the as-is BIM generated from the data before using them for making decisions about facilities. This paper presents the data and as-is BIM QA requirements of civil engineers and demonstrates that the required QA information can be derived by analyzing the patterns in the deviations between the data and the as-is BIMs. We formalized this idea as a deviation analysis method for efficient and effective QA of the data and as-is BIMs. An evaluation of results obtained through this approach shows the potential of this method for achieving timely, detailed, comprehensive, and quantitative assessment of various types of data/model quality issues.
\end{abstract}

Keywords: Building Information Modeling; Laser Scanning; Quality Assessment

\section{INTRODUCTION}

Laser scanning is a method for capturing detailed geometries of constructed facilities and constructing as-is Building Information Models (BIMs) (Tang et al. 2010). These as-is BIMs can serve as central project knowledge bases for various applications, such as facility management and renovation design (Tang et al. 2010). In supporting such applications, it is critical to conduct timely, detailed, and comprehensive quality assessments (QA) of the laser-scanned data and as-is BIMs. Efficient QA can help to reduce the project delay and improve the proactivity of the decision making. Detailed and comprehensive quality information about the data and the as-is models is necessary for enabling better use of the data and models.

One QA method that has been utilized by service providers is the physical measurement method (Cheok et al. 2009; Cheok and Franazsek 2009). In this method, engineers take a number of physical measurements in the facility and compare them 
to the corresponding virtual measurements in the as-is BIM. These measurements can be performed randomly to estimate the confidence that a 2D/3D building plan meets a given accuracy requirement based on statistical analysis of the differences between a number of virtual and physical measurements. While effective, this method suffers from additional time needed for data collection and analyses. Collecting physical measurements is laborious and time-consuming, resulting in evaluation periods of days or even weeks (Anil et al. 2011; Cheok and Franazsek 2009). Such tedious measurement collections compromise the timeliness of QA and pose accessibility issues for some parts of the facility (Anil et al. 2011). Another limitation of this method is that it highlights the error, but does not provide an assessment of possible reasons for the error. For example, using this method, an engineer has limited clues about whether the inconsistencies are caused by mistakes made by the modeler or by a scanner calibration problem. A final limitation of this approach is that, in practice, it is impractical to physically measure every possible location; hence, it is likely to miss some problematic data points or model parts.

To overcome the limitations of the physical measurement method and achieve timely, detailed, and comprehensive QA of laser-scanned data and as-is BIMs, we have formulated a deviation analysis based approach. Assuming that most parts of an as-is BIM derived from the data align well with the data, any substantial deviations between the data and the BIM indicate potential quality issues of the data and BIM. Similarly, data collected at different locations for the same facility should agree with each other in overlapping regions, and substantial deviations between scans collected at different stations for the same objects could serve as indicators of data quality issues. According to our investigations, different sources and types of errors in the data or model lead to different deviation patterns. As a result, these deviation patterns can be visualized and can guide engineers in identifying potential data/model quality issues. Figure 1 shows a colorized deviation pattern between the data and the BIM of a building's roof.

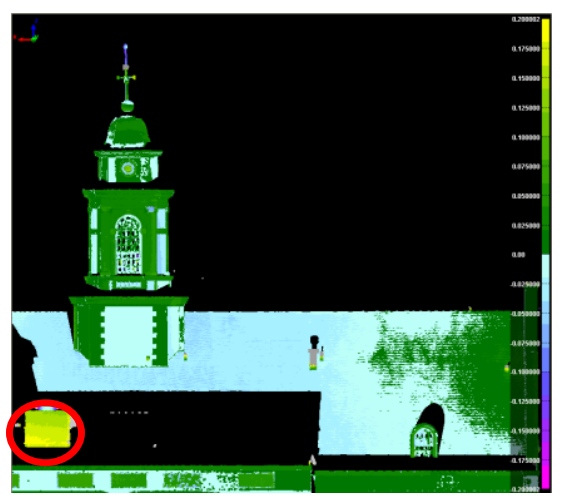

Figure 1. Deviation patterns color coded for a roof of a building: in the circle at the bottom left, an object has larger deviations (yellow) compared with the other parts of the roof (blue and green). Such deviation patterns can guide engineers to investigate and analyze that part of the model and data in depth.

The deviation analysis method addresses the limitations of the physical measurement method. It does not require physical measurements; hence, it can 
deliver timely data and model quality information. Classifications of deviation patterns enable engineers to identify different types of quality issues with the data and the model. For all areas covered by data, this method can conduct comprehensive QA. This paper presents the data and as-is BIM QA requirements of civil engineers, the deviation analysis method for QA, and evaluation results illustrating how this deviation analysis method meets the domain requirements.

\section{RELATED STUDIES}

Previous studies have explored a physical measurement method for the QA of as-is 2D/3D building plans (Cheok et al. 2009; Cheok and Franazsek 2009). While this approach is effective in identifying modeling issues, it is time consuming and difficult to achieve a comprehensive assessment of the model quality since it directly compares the model with the physical measurements. In addition, it focuses only on QA of 2D/3D models and does not address the QA of the data.

Previous studies in multiple domains have explored methods for generating deviation patterns between the data and models for quality control of manufactured mechanical parts or constructed facilities (B. Akinci et al. 2006; Gordon et al. 2003). For the quality control of mechanical parts, the manufacturing industry uses 3D reverse engineering software for generating the deviations of the actual geometries of these parts from the designed geometries (Innovmetric, Inc. 2010). Compared with the QA of a building project, the QA of mechanical parts occurs in a controlled environment for relatively small objects with minimal occlusion. In the domain of construction management, researchers generated and visualized the deviations between an as-built model derived from laser-scanned data and an as-designed BIM for detecting and managing construction defects (B. Akinci et al. 2006). Since these studies focused on quality control of physical objects rather than quality control of 3D data and models, they conducted limited explorations about the QA of data and models and how to identify types of data/model errors based on deviation patterns.

Another method relevant to deviation analysis is clash detection - a method used by building renovation projects for detecting the spatial conflicts of the designed and existing objects (Autodesk Inc. 2010). This method identifies locations where the space between designed and as-is objects is negative (strict physical clashes) or smaller than user-specified tolerances (soft clashes). Binary clash/non-clash information is a type of deviation indicator, but its binary nature poses limitations when detailed deviation patterns, rather than binary clash maps, are needed.

\section{QUALITY ASSESSMENT REQUIREMENTS AND AS-IS BIM WORKFLOW}

Two major domain requirements need to be addressed for achieving timely, detailed, and more comprehensive QA of laser-scanned data and as-is BIMs. First, various quality issues of the data and models occur along the workflow of constructing an as-is BIM, and engineers need to pinpoint the types of these issues so that they can fix them or make decisions with awareness of the identified errors exist in the model. For instance, engineers need to know whether large deviations between overlapping scans are caused by scanner calibration problems or data registration 
errors, so that they can recalibrate the scanner or improve data registration accordingly. Second, most applications have specific tolerances about the accuracy of the data and as-is BIMs. The engineers need to quantify the magnitudes of deviations or errors. For instance, if an architect specifies that the positioning accuracy tolerance for windows is $5 \mathrm{~cm}$, then the QA method should enable the architect to identify all locations having errors larger than $5 \mathrm{~cm}$.

A typical as-is BIM construction workflow is composed of three phases: 1) Data collection; 2) Data preprocessing; and 3) Modeling the BIM. More detailed descriptions of these three steps can be found in (Tang et al. 2010). Generally, the first two phases influence the data quality, while the last phase influences the model quality. The major error sources in the data collection phase include: 1) Incorrect calibration of the scanner; 2) Mixed pixels due to spatial discontinuity edges; and 3) Range errors due to specular reflections (Anil et al. 2011). Data preprocessing mainly involves identifying and removing noisy data points, and aligning multiple scans in local coordinate systems to a common coordinate system (known as data registration). The major error sources involved in this step include: 1) Incorrect noise removals; and 2) Data registration errors. The major error sources in the modeling phase include: 1) Failing to model physical components; 2) Modeling components using incorrect shapes; 3) Modeling components with incorrect position. A QA approach should ideally be able to identify all these types of quality issues and to enable engineers to quantify and understand their implications to the domain applications. Due to the space limits, this paper focuses on the domain requirements and an evaluation of the deviation analysis method on satisfying these requirements without detailing data processing steps and the definitions of all error types. More details on these aspects can be found in (Anil et al. 2011).

\section{DEVIATION ANALYSIS}

The deviation analysis method completes the QA in two steps: 1) Deviation computation and 2) Deviation visualization. First, an algorithm computes the deviations of data points from the surfaces of the as-is BIM based on the assumption that all data points and the as-is BIM are in the same coordinate system. This assumption is valid for all projects studied in this research. In these projects, engineers first registered the laser-scanned point clouds to a geographic coordinate system, and then created BIM in that coordinate system. The deviations can be computed in several ways. The most common way is to compute the minimum Euclidian distance from each point to its nearest surface in the BIM. Other methods include computing the point-surface distances along user-specified directions, such as the $\mathrm{X}, \mathrm{Y}, \mathrm{Z}$ direction of the common coordinate system or the direction of the surface normal. In this paper, we tested the approach using the minimum Euclidian distances.

After generating the deviations, engineers visualize the deviation patterns. Generally, they can configure several aspects of the visualization algorithms. First, they can configure the color maps. Two major categories of color maps are the continuous color map and the binary color map. In this paper, we focus on evaluating a red-yellow continuous color map (gradual color variation from red to yellow with the reduction of deviation values) and a yellow-green binary color map (assign 
yellow/green color to data or model with deviations larger/smaller than a user-specified threshold), as detailed later. Second, for continuous color maps, engineers can configure it as unsigned or signed. Unsigned color maps visualize the absolute deviation values, so that deviations of the same absolute values will have the same color, while signed color maps visualize equivalent positive and negative deviations with different colors. This paper focuses on signed color maps, which we found to be more effective in practice. Third, engineers can configure the scale of the color map so that they can control which ranges of deviations are of interest. Specifically, they can configure the maximum and minimum deviation values visualized; they can also set the threshold value for the binary color map to only distinguish deviations larger and smaller than that threshold. Finally, engineers can choose to colorize points or colorize the BIM surfaces. In this paper, we focus on evaluating the point colorization method, since it can give more detailed and localized deviation information for QA (Anil et al. 2011).

In addition to deviation generation and visualization, statistical analysis can be used to analyze the deviation patterns. One example is to create the deviation histograms for a certain region for obtaining the mode of deviation values, as shown in (Anil et al. 2011). Such statistical methods could make the deviation pattern analysis automatic. This paper focuses on the deviation generation and visualization, and leaves the automated deviation analysis for future exploration.

\section{EVALUATION RESULTS}

We have evaluated the technical feasibility of the developed QA approach by using data and models generated for several projects by service providers. In this paper, we use data from one of these projects to illustrate the potential effectiveness of the deviation analysis method. Specifically, we conducted two sets of evaluations and analyzed the results for understanding how this method addresses the two domain requirements of 1) identifying different types of data and model quality issues and 2) quantifying the magnitudes of these issues. To investigate whether the deviation analysis can help engineers to identify different types of quality issues of the data and as-is BIMs, we generated and analyzed large amounts of deviation patterns. We found that all studied types of data/model quality issues can produce distinguishable deviation patterns, and these patterns can serve as indicators for guiding engineers in pinpointing the error types and sources.

Figure 2 shows typical deviation patterns revealing various data quality issues. Figure 2(a) shows the top view of one of the studied buildings. It uses a binary color map to highlight parts of the roof with deviations larger than $2.5 \mathrm{~cm}$ as yellow. On the roof, two circular stripes are centered around two scanning locations on the platform. These abnormal patterns correlated with the scanning locations indicate the likelihood of incorrect scanner calibration. Figure 2(b) shows deviation patterns on the front façade of this building using a continuous color map. On the roof, the deviations increase roughly linearly from left to right. According to detailed analysis, this gradient deviation pattern is caused by an inaccurate rotation angle used for data registration. Figure 2(c) shows the deviation patterns around a window on the façade of this building, using the same binary map adopted in 2(a). The deviations around 
the two vertical edges of a window are all larger than $2.5 \mathrm{~cm}$. Detailed investigations revealed that the mixed pixels around spatial discontinuities influence the data quality and cause such patterns. Using the same binary color map, Figure 2(c) and (d) show that for all specular objects with high reflectivity, such as window glass and the metallic awning, the deviations are larger than other parts, likely due to higher noise in these regions. These observed correlations between deviation patterns and types of data quality issues show the effectiveness of the deviation analysis method for pinpointing types of data problems.

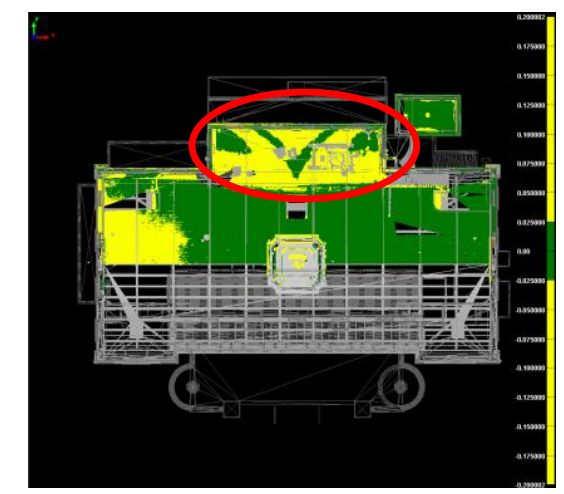

(a) Potential scanner calibration problem

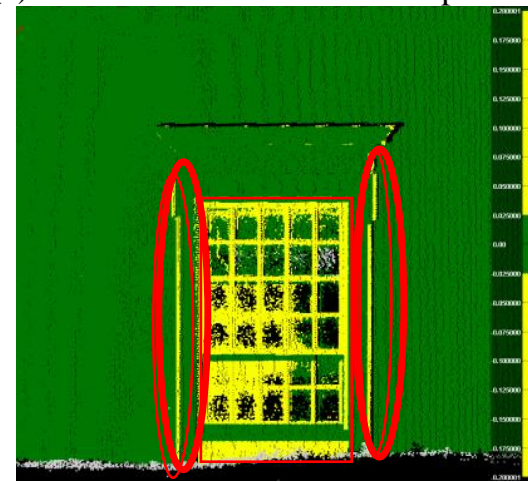

(c) Mixed pixels at spatial discontinuities

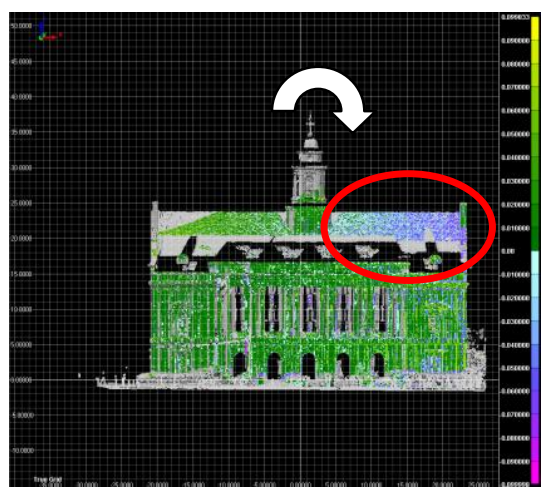

(b) A rotation error in data registration

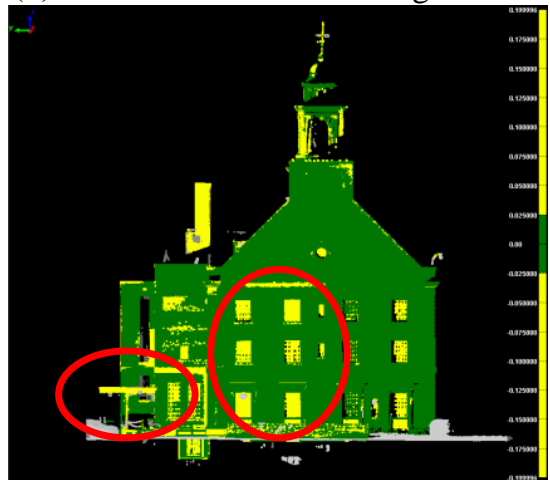

(d) Low data quality on specular surfaces

Figure 2 Deviation patterns for identifying various data quality issues

Figure 3 shows deviation patterns caused by various modeling errors on one of the studied buildings. Figure 3(a) shows the photo of a part of the façade, and 3(b) shows the deviation pattern of this region based on the continuous color map. Figure 3(b) shows a rectangular region with large deviations. That pattern is caused by an inset rectangular region on the wall, which was a window but was sealed with bricks that the modeler failed to model. Figure 3(c) shows abnormal patterns on all columns. The radii of these columns vary parabolically, while the modeler assumed linear variations. This example shows how to use the deviation patterns for identifying problems of modeling with incorrect shapes. Figure 3(d) shows the deviation patterns on another part of the façade using continuous color map. Different colors on the first and second floors indicate that these walls are not coplanar (around $2 \mathrm{~cm}$ misalignment), while the modeler assumed that they were. All these examples indicate that the deviation analysis method can pinpoint various model quality issues.

In relation to the requirement about quantifying the data/model quality issues, 
the deviation analysis method enables engineers to configure parameters of the color maps for visualizing deviations of interest. First, engineers can configure the maximum and minimum deviations visualized by a continuous color map to only show the patterns within that range based on their requirements. In Figure 2(b), the range of interest is $(-0.1 \mathrm{~m}$ to $0.1 \mathrm{~m})$. In Figure $3(\mathrm{~b})$, (c), and (d), the ranges of interest are $(-0.2 \mathrm{~m}$ to $0.2 \mathrm{~m}),(-0.05 \mathrm{~m}$ to $0.05 \mathrm{~m})$, and $(-0.05 \mathrm{~m}$ to $0.05 \mathrm{~m})$ respectively. Generally, identifying "failing to model physical components" issues needs a larger range than identifying the other two types of modeling issues, since missing a component typically causes relatively larger deviations. Similarly, for the binary color map, engineers can configure the threshold to only highlight regions exceeding a tolerance. According to the tolerance specified in the project requirements, we used $0.025 \mathrm{~m}$ as the threshold for all shown results.

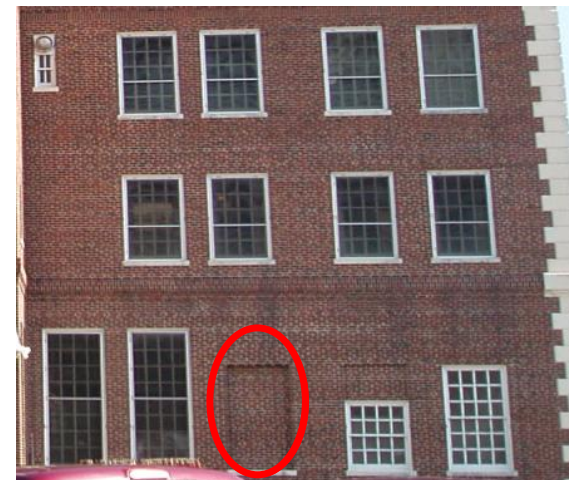

(a) Photo of a part of the back façade

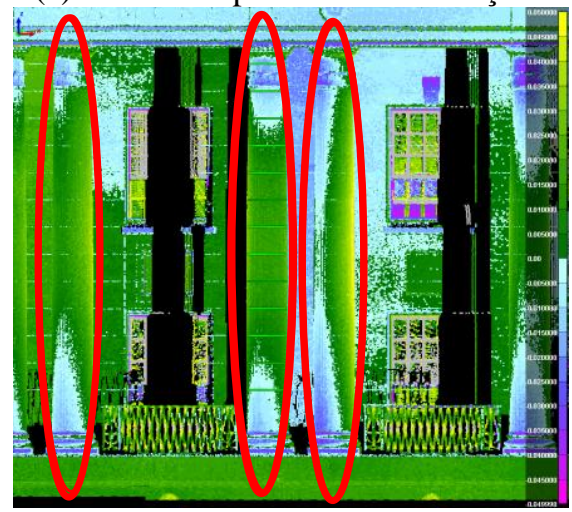

(c) Model using incorrect shape

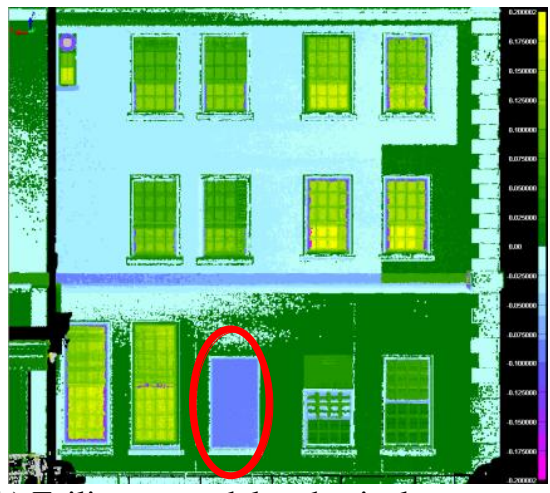

(b) Failing to model a physical component

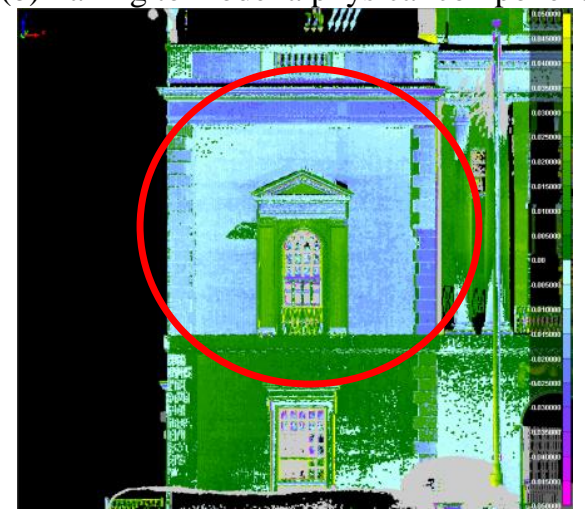

(d) Model components with incorrect positions

Figure 3 Deviation patterns for identifying various model quality issues

\section{SUMMARY AND FUTURE RESEARCH}

In this paper, we formulated a deviation analysis method to overcome the limitations of the physical measurement method for the QA of laser-scanned data and as-is BIMs. We illustrated the method's effectiveness on addressing the domain requirements of timely, detailed, and comprehensive QA of the data and BIM. Based on a list of data and as-is BIM quality issues that we identified, we found that this deviation analysis method can detect all listed quality issues. This method also enables engineers to quantify and visualize the deviations of certain magnitude for 
improving their quantitative awareness of the data and BIM quality issues.

In the future, we plan to improve this method in these aspects: 1) identify more types of data and model quality issues and further evaluate the performance of the deviation analysis method on identifying them; 2) formulate a taxonomy of data and quality issues for formalized and systematical QA of 3D data and as-is BIMs; 3) conduct more detailed evaluation of the efficiency of this method; and 4) develop pattern recognition methods for automated deviation pattern analysis. In addition to these technological improvements, we envision that this approach will evolve into a methodology for automated data and model quality management to aid data driven decision making in construction and facility management projects, and to aid data/model quality driven data collection and interpretation on job sites.

\section{ACKNOWLEDGEMENTS}

This material is based upon work supported by the U.S. General Services Administration under Grant No. GS00P09CYP0321. Any opinions, findings, conclusions, or recommendations presented in this publication are those of authors and do not necessarily reflect the views of the U.S. General Services Administration.

\section{REFERENCES}

Akinci, B., Boukamp, F., Gordon, C., Huber, D., Lyons, C., and Park, K. (2006). “A formalism for utilization of sensor systems and integrated project models for active construction quality control." Automation in Construction, Elsevier, 15(2), 124-138.

Anil, E. B., Tang, P., Akinci, Burcu, and Huber, Daniel. (2011). “Assessment of Quality of As-is Building Information Models Generated from Point Clouds Using Deviation Analysis." Proceedings of SPIE, San Jose, California, USA. Autodesk, Inc. (2010). "Navisworks." http://usa.autodesk.com/adsk/servlet/pc/index?siteID=123112\&id=10571060.

Cheok, G. S., Filliben, J. J., and Lytle, A. M. (2009). Guidelines for accepting 2D building plans. NIST Interagency/Internal Report (NISTIR) - 7638.

Cheok, G. S., and Franazsek, M. (2009). Phase III: Evaluation of an Acceptance Sampling Method for 2D/3D Building Plans. NIST Interagency/Internal report (NISTIR)-7659.

Gordon, C., Boukamp, F., Huber, D., Latimer, E., Park, K., and Akinci, B. (2003).

"Combining reality capture technologies for construction defect detection: a case study." EIA9: E-Activities and Intelligent Support in Design and the Built Environment, 9th EuropIA International Conference, Citeseer, 99-108.

Innovmetric, Inc. (2010). "Polyworks v11.0." www.innovmetric.com. Tang, P., Huber, Daniel, Akinci, Burcu, Lipman, R., and Lytle, A. (2010).

"Automatic reconstruction of as-built building information models from laser-scanned point clouds: A review of related techniques." Automation in Construction, 19(7), 14. 